\title{
Fragmente der Erinnerung: Die Historiographie von Montecassino 9. bis 11. Jahrhundert
}

Viele Beiträge dieses Bandes beschäftigen sich mit Überlieferungsfragmenten; aus einem ursprünglich Ganzen ist nur ein Teil auf uns gekommen, sei es bewusst durch Selektion oder durch Verlust. Hier geht es hingegen um Text-Fragmente, die bereits in dieser Form in Handschriften eingetragen wurden. Dabei waren diese Codices von der Anlage her nicht als Sammlung von Auszügen oder Bruchstücken, also etwa als Florilegien, konzipiert. Der fragmentarische Charakter mancher eingetragener Texte ergab sich auch nicht immer aus ihrer Position in der Handschrift (etwa als Nachträge, Glossen oder Randvermerke), sondern manche wurden durchaus geplant im fortlaufenden Textfluss eingetragen. Es handelte sich also um eine Art von ,écriture fragmentaire', freilich nicht in ästhetischer, sondern wohl in durchaus pragmatischer Absicht. Die Texte, um die es hier gehen soll, stammen aus der „Werkstätte der Erinnerung“ von Montecassino. ${ }^{1}$ Meine 2001 erschienene Monographie zu diesem Thema beruhte vor allem auf der Untersuchung dreier süditalienischer Handschriften aus dem Mittelalter, je einer aus der Biblioteca Apostolica Vaticana (Vat.lat. 5001, um 1300) und aus den Klosterarchiven von Montecassino (Cod. 175, 10. Jh.) und Cava de' Tirreni (Cod. 4, um 1000). ${ }^{2}$ Auf den ersten Blick hat nur einer dieser drei Codices etwas mit Montecassino zu tun; doch gelang es, für die Handschrift aus Cava einen Ursprung in Montecassino, für die aus der Vaticana eine indirekte Vorlage aus Montecassino sehr wahrscheinlich zu machen. Alle drei Handschriften waren daher Zeugnisse für die Schriftlichkeit und den Umgang mit der Vergangenheit vom Ende des 9. bis zum Beginn des 11. Jahrhunderts.

Das war eine schwierige Zeit für die Mönchsgemeinschaft, die ihren Ursprung auf den heiligen Benedikt zurückführte. Nach der Zerstörung durch die Langobarden gegen Ende des 6. Jahrhunderts war Montecassino am Beginn des 8. Jahrhunderts wieder gegründet worden und hatte sich rasch zu einem der reichsten und einflussreichsten Klöster der Zeit entwickelt. ${ }^{3}$ Um 790 hatte hier der vielseitige Gelehrte Paulus Diaconus seine Langobardengeschichte geschrieben. Seit Mitte des 9. Jahrhunderts wurde Süditalien aber von immer unübersichtlicheren Regionalkonflikten erschüttert. ${ }^{4}$ Im September 883 wurde Montecassino von sarazenischen Plünderern zerstört, und die Mönche zogen sich nach Teano zurück. ${ }^{5}$ Doch hier wurde die Klostergemeinschaft von einer weiteren Katastrophe betroffen, einem Brand, der wohl im Jahr 896 einen Teil des

${ }^{1}$ Walter PoHL, Werkstätte der Erinnerung. Montecassino und die Gestaltung der langobardischen Vergangenheit (Mitteilungen des Instituts für Österreichische Geschichtsforschung, Erg. Bd. 39). Wien 2001.

2 Ausführlich POHL, Werkstätte (wie Anm. 1); siehe auch DERS., History in fragments. Montecassino's politics of memory, in: Early Medieval Europe 10/3 (2001) 343-374; DERS., Testi e identità in manoscritti cassinesi dei secc. IX-XI, in: Flavia DE RUBEIS und Walter POHL (Hg.), Le scritture dai monasteri. Atti del II $^{\circ}$ Seminario Internazionale di Studio ,I Monasteri nell'Alto Medioevo‘ (Acta Instituti Romani Finlandiae 29). Rom 2003, 197-206.

${ }^{3}$ Faustino Avagliano (Hg.), Montecassino dalla prima alla seconda distruzione. Momenti e aspetti di storia cassinese secc. VI-IX (Miscellanea Cassinese 55). Montecassino 1987.

${ }^{4}$ Barbara Kreutz, Before the Normans. Southern Italy in the Ninth and Tenth Centuries. Philadelphia 1991; I Longobardi dei ducati di Spoleto e Benevento. Atti del XVI congresso internazionale di studi sull'alto medioevo. Spoleto 2003.

5 Erchempert, Historia Langobardorum Beneventanorum c. 44, hg. von Georg WAITZ (MGH SS rerum Langobardicarum et Italicarum 1). Hannover 1878, 234-264, hier 251; Chronica monasterii Casinensis, hg. von Hartmut HoffMANN (MGH SS 34). Hannover 1980, 114 (jeweils datiert mit 884). Zur Datierung 883 Hartmut HoffMANN, Die älteren Abtslisten von Montecassino, in: Quellen und Forschungen aus italienischen Archiven und Bibliotheken 47 (1967) 224-354, hier 261. Dazu und zum Folgenden PoHL, Werkstätte (wie Anm. 1) 154-162. 
Schatzes und des Archivs vernichtete. ${ }^{6}$ Bald darauf übersiedelten die Mönche weiter nach Capua, wo sie unter die Kontrolle der dortigen Fürsten gerieten und damit direkt in die wechselvollen politischen Auseinandersetzungen der Zeit verwickelt wurden. Erst allmählich gelang im 10. Jahrhundert die Rückübersiedlung nach Montecassino. Dort folgte in der 2. Hälfte des 11. Jahrhunderts eine Blütezeit des Klosters und seines Skriptoriums, die mit dem Namen des Abtes Desiderius verknüpft ist. ${ }^{7}$ Im Jahr 1099 begann schließlich Leo von Ostia die große Klosterchronik, die über die wechselhafte Geschichte Montecassinos Auskunft gibt. $^{8}$ In einer aufwändigen Montage von Erzählungen und Urkunden repräsentiert diese ,ChartularChronik', ähnlich wie es um dieselbe Zeit in San Vincenzo al Volturno, S. Sofia in Benevent oder Farfa geschah, ein großes ,institutionelles Narrativ ${ }^{6}$, in dem die fundierende Vergangenheit systematisch dargelegt wurde.

Ähnliches gab es im 9.-11. Jahrhundert in Montecassino offenbar nicht; große Geschichtswerke wie das des Paulus Diaconus oder des Leo von Ostia sucht man hier in jenen Jahrhunderten vergebens. Doch produzierte das Skriptorium weiterhin beachtliche Texte und Handschriften. Darunter ist die bald nach 920 niedergeschriebene älteste in Montecassino erhaltene Regelhandschrift, heute Codex Casinensis $175 .{ }^{9}$ Neben der Benedikt-Regel mit dem Kommentar Hildemars aus dem 9. Jahrhundert enthält diese Handschrift eine Reihe kleinerer Texte, die wiederum den Bezug zu den anderen beiden Codices herstellen. Auf Grund dieser textlichen Parallelen lässt sich auch das in Cava de' Tirreni erhaltene und in den ersten Jahren nach 1000 geschriebene repräsentative langobardische Rechtsbuch (der Codex Cavensis 4), ebenfalls ergänzt durch zahlreiche kleinere Texte, mit großer Wahrscheinlichkeit aus Montecassino herleiten. ${ }^{10}$ Beide Handschriften sind wiederum vielfach textlich verknüpft mit einer Sammelhandschrift, die erst um 1300 in Salerno geschrieben wurde, dem Vaticanus lat. 5001. ${ }^{11}$ Dabei handelt es sich um die einzige Überlieferung wesentlicher historiographischer Werke aus dem langobardischen Süditalien, nämlich der Historia des Cassineser Mönchs Erchempert vom Ende des 9. Jahrhunderts und des Chronicon Salernitanum, 974 in Salerno verfasst. ${ }^{12}$ Ein Vermerk am Beginn des Codex weist darauf hin, dass er von einem älteren, in Beneventana (litterae Langobardorum) geschriebenen Geschichtsbuch (liber quarundam ystoriarum) kopiert war; dieses langobardische Geschichtsbuch enthielt das Chronicon Salernitanum und wurde daher irgendwann nach 974 in Salerno geschrieben. Fast alle der zahlreichen weiteren Texte im Vaticanus gehen auf eine noch ältere Vorlage zurück, die kurz vor 900 in Montecassino entstanden sein muss. ${ }^{13}$

Es sind die kleinen, oft fragmentarischen Texte in den drei Handschriften, die einen außergewöhnlichen Einblick in die Erinnerungskultur der Klostergemeinschaft in jenen schwierigen Zeiten ermöglichen. Reizvoll ist gerade ihre Vielfalt; neben kleineren Chroniken und Fürstenlisten finden sich Gedichte und Epitaphien, Verträge und Glossarien des Langobardenrechtes, Briefe und Verzeichnisse. Viele dieser Texte las-

\footnotetext{
${ }^{6}$ Chronica monasterii Casinensis (wie Anm. 5) 126f.; Walter PoHL, Anstrengungen des Erinnerns. Schreiben in Montecassino nach der zweiten Zerstörung (883), in: Christoph Dartmann, Thomas ScharfF und Christoph F. Weber (Hg.), Zwischen Pragmatik und Performanz. Dimensionen mittelalterlicher Schriftkultur (Utrecht Studies in Medieval Literacy 18). Im Druck.

${ }^{7}$ Herbert E. J. COwDREY, The Age of Abbot Desiderius. Montecassino, the Papacy and the Normans in the Eleventh and Early Twelfth Centuries. Oxford 1983; Faustino Avagliano und Oronzo PeCERE (Hg.), L'età dell'abbate Desiderio III/1: Storia, arte e cultura. Atti del IV ${ }^{0}$ Convegno di studi sul Medioevo meridionale. Montecassino 1992; Francis NewTON, The Scriptorium and Library at Monte Cassino 1058-1105. Cambridge 1999; Graham A. Loud, Montecassino and Benevento in the Middle Ages (Variorum Collected Studies Series 673). Aldershot-Burlington 2000.

${ }^{8}$ Chronica monasterii Casinensis (wie Anm. 5); Hartmut Hoffmann, Studien zur Chronik von Montecassino, in: Deutsches Archiv 29 (1973) 59-162.

${ }^{9}$ PoHL, Werkstätte (wie Anm. 1) 77-107.

10 Zur Begründung dieser These PoHL, Werkstätte (wie Anm. 1) 108-151.

11 PoHL, Werkstätte (wie Anm. 1) 14-76.

12 Chronicon Salernitanum. A Critical Edition with Studies on Literary and Historical Sources and on Language, hg. von Ulla Westerbergh (Acta Universitatis Stockholmiensis, Studia Latina Stockholmiensia 3). Stockholm-Lund 1956; Nicola CiLENTO, La tradizione manoscritta di Erchemperto e del ,Chronicon Salernitanum', in: DERS., Italia meridionale longobarda. MilanoNapoli ${ }^{2}$ 1971, 73-102; Massimo OldONI, Anonimo Salernitano del X secolo. Napoli 1972; Huguette TAVIANI-CAROZZI, La principauté Lombarde de Salerne, IX $-\mathrm{XI}^{\mathrm{e}}$ siècle. 2 Bde. (Collection de l'École Française de Rome 152). Rome 1991.

13 PoHL, Werkstätte (wie Anm. 1) 15-18.
} 
sen sich auf zur Zeit der Kompilation aktuelle Interessen der Klostergemeinschaft beziehen. Hier möchte ich mich aber auf die im engeren Sinn historiographischen Bemühungen der Mönche konzentrieren. Zum Teil entstanden dabei längere und einigermaßen kohärente Texte, zum Beispiel die Continuatio Casinensis der Langobardengeschichte des Paulus Diaconus ${ }^{14}$ sowie die Historia Erchemperts, beide in der vatikanischen Handschrift überliefert. Die bis 889 reichende Erchempert-Chronik ist nicht nur der längste der historiographischen Texte aus Montecassino - knapp 30 Seiten in der Monumenta-Edition - sondern auch der einzige, dessen Autor wegen seiner persönlichen und meist sehr zeitkritischen Bemerkungen namentlich bekannt ist. Deutlich wird darin, dass Erchempert in seiner Chronik die Ereignisse fortlaufend niedergeschrieben haben muss, denn seine Haltung zum Comes und späteren Princeps Atenulf I. von Capua ändert sich im Lauf des Textes diametral. ${ }^{15}$

Viel inhomogener ist jener Abschnitt aus der Cassineser Handschrift, den Waitz in den MGH als Chronica Sancti Benedicti Casinensis ediert hat. ${ }^{16}$ Es handelt sich um eine historiographische Kompilation aus der Zeit, als Kaiser Ludwig II. auf einem Zug gegen die Sarazenen 867 nach Montecassino kam. Sie enthält zumeist knappe Informationen über die Geschichte des Klosters und seiner Äbte, ergänzt durch Nachrichten aus der Langobardengeschichte des Paulus Diaconus. Zwischen diesen bruchstückhaft angeordneten Elementen findet sich auch ein ausführlicher Bericht vom Kriegszug Ludwigs II. sowie eine Abschrift des Kapitulars, mit dem er die Modalitäten des Zuges detailliert geregelt hatte. Es ist gut möglich, dass dieser heterogene Text bereits in einer ca. 867 entstandenen Vorlage der Regelhandschrift zu finden war. Er endet mit einer noch einige Jahre lang ergänzten Tabelle, in der die Amtszeiten der Cassineser Äbte mit denen der Fürsten von Benevent und später Salerno, ab der Mitte des 9. Jahrhunderts auch der Emire von Bari und der fränkischen Herrscher des Regnum Italiae synchronisiert werden, ähnlich den fila regnorum der HieronymusChronik. ${ }^{17}$ In der ein gutes halbes Jahrhundert später angelegten Cassineser Handschrift wird kein Versuch unternommen, an die chronikalischen Abschnitte oder an die Abtliste anzuknüpfen. Sie stellt danach Kataloge der Päpste, der römischen und byzantinischen Kaiser, der Langobardenkönige und der Fürsten von Benevent jeweils von den Anfängen bis in die 920er Jahre. ${ }^{18}$

Noch fragmentarischer sind jene Bruchstücke über die Geschichte der Grafen von Capua, die sich in unterschiedlicher Zusammenstellung sowohl in der Handschrift von Montecassino als auch von Cava finden. Sowohl Pertz als auch Waitz haben ein Amalgam beider Fassungen als Catalogus comitum Capuae herausgegeben. ${ }^{19}$ Das Chronicon comitum Capuae, das Pertz edierte, ist hingegen eine Fälschung von Francesco Maria Pratilli aus dem 18. Jahrhundert. ${ }^{20}$ Fragmente fordern zuweilen Fälschungen heraus. Beim Text unserer Handschriften handelt es sich um eine knappe Übersicht über die Grafen von Capua vom Beginn des 9. Jahrhunderts bis zum Tod Atenulfs I. (909); ein weiteres Bruchstück auf der letzten Seite des Casinensis fasst Kriegsereignisse zwischen 903 und 922 (den Ungarnzug in Apulien) zusammen. Siebzig Jahre später wurde in den freien Raum am Ende des Hauptteils ein seltsamer Bericht von der Ermordung des Landenulf von Capua im Jahr 993 eingetragen. Der Fürst soll von seinen Mördern nackt auf der Piazza liegengelassen worden sein; Abt Manso sorgte dafür, dass er in Montecassino vor der Sakristei begraben wurde. Angeblich

14 Continuatio Casinensis. Pauli Continuationes, hg. von Georg WAITZ (MGH SS rerum Langobardicarum et Italicarum 1). Hannover 1878, 198-200.

15 PoHL, Werkstätte (wie Anm. 1) 33-42.

${ }^{16}$ Chronica Sancti Benedicti Casinensis, hg. von Georg WAITZ (MGH SS rerum Langobardicarum et Italicarum 1). Hannover 1878, 468-488; Chronica Sancti Benedicti Casinensis, hg. von Andrea BerTo. Firenze 2006.

17 Walter PoHL, Trasmissione e contesto delle Chronicae Sancti Benedicti Casinensis: il Codex Casinensis 175, in: Chronica Sancti Benedicti Casinensis, hg. von BERTO (wie Anm. 16) XXX-XLI.

${ }^{18}$ Nur die Liste der Langobardenkönige endet mit der fränkischen Eroberung 774; eine Liste der fränkischen Herrscher im Regnum Italiae fehlt, was aus süditalienischer Sicht in den 920er Jahren nicht überrascht.

19 Comites Capuae, hg. von Georg Heinrich PERTz (MGH SS 3). Hannover 1839, 205f. = Catalogus comitum Capuae, hg. von Georg WAITZ (MGH SS rerum Langobardicarum et Italicarum 1). Hannover 1878, 498-501; Nicola CiLENTO, La cronaca della dinastia capuana, in: DERS., Italia meridionale longobarda (wie Anm. 12) 103-174.

${ }^{20}$ Chronicon comitum Capuae, hg. von Georg Heinrich PerTZ (MGH SS 3). Hannover 1839, 207-210; Nicola CILENTO, Il falsario della storia dei Longobardi meridionali: Francesco Maria Pratilli (1689-1763), in: DERS., Italia meridionale longobarda (wie Anm. 12) 24-39. 
sollen sich an seinem Grab bald Wunder ereignet haben. Abt Manso war 985 auf Initiative von Landenulfs politisch höchst aktiver Mutter Aloara eingesetzt worden und stand in den Parteiungen jener Jahre auf der Seite des Fürsten. ${ }^{21}$ Die Nachricht über die Wunder an dessen Grab ist auffallender Weise der einzige Wunderbericht und einer der ganz wenigen Texte hagiographischen Charakters in den drei Handschriften. Das ist charakteristisch für den wenig heiligmäßigen, aber sehr politisch-pragmatischen Stil der Äbte jener Zeit. Derselbe Bericht wurde bald darauf auch in die Handschrift von Cava übernommen, eines der untrüglichen Zeichen, dass auch sie in Montecassino entstanden sein muss.

Derartige Textfragmente, die sich in zwei oder allen drei Handschriften, teilweise auch mehrmals finden, sind besonders aufschlussreich für die historiographischen Interessen der Klostergemeinschaft. Eines der interessantesten Beispiele ist die Geschichte von Pippins Bruder Karlmann. ${ }^{22}$ Im Liber Pontificalis findet sich die Nachricht, dass er nach seinem Verzicht auf die Herrschaft (747) nach Rom zu Papst Zacharias gekommen und bald darauf in die Mönchsgemeinschaft von Montecassino eingetreten sei. ${ }^{23}$ Im Konflikt mit Papst Stephan II. und Pippin sandte ihn aber der Langobardenkönig Aistulf 754 ins Frankenreich, wo er in ein Kloster gesteckt wurde und bald darauf starb. Die in der vatikanischen Handschrift erhaltene Continuatio des Paulus Diaconus übernimmt diese Nachricht mit zwei wesentlichen Veränderungen. ${ }^{24}$ Erstens wird Carlomannus durch den bekannteren Carlomagnus, Karl den Großen, ersetzt. Zweitens berichtet ein Zusatz, der Körper des Verstorbenen sei in einem goldenen edelsteinbesetzten Kästchen, in locello aureo gemmato, nach Montecassino gesandt worden. Weiter zugespitzt wird diese Nachricht in einem Zusatz zur langobardischen Königsliste im Cassineser Codex, wo auch noch der Konflikt mit dem Papst geglättet ist: Karlmann sei als Gesandter des Papstes und Repräsentant der römischen Res Publica nach Gallien gegangen. ${ }^{25}$ Im 10. Jahrhundert war man in Montecassino also stolz darauf, das Grab Karls des Großen zu besitzen, woran sich eine Reihe von frommen Legenden über die Zeit des großen Kaisers als Mönch knüpften, wie sie im Chronicon Salernitanum überliefert sind. ${ }^{26}$ Noch Leo von Ostia übernahm nach 1100 die Version der Continuatio, während in der Handschrift von Cava nur von Carlomannus die Rede ist. ${ }^{27}$

Entscheidende identitätsstiftende Überlieferungen der Klostergemeinschaft sind hier also in sehr verknappter Form erhalten. Es ging ja auch nicht darum, Außenstehende zu überzeugen, sondern an Bekanntes zu erinnern. Ähnlich verfuhr man mit der Nachricht von der monastischen Konversion des Langobardenkönigs Ratchis $749 .{ }^{28}$ Im Bericht des Liber Pontificalis ist Montecassino nicht einmal erwähnt. Wieder ist es der Continuator des Paulus, der diese Information ergänzt und dazu die Nachricht von der Stiftung und großzügigen Dotierung des Frauenklosters Plumbariola, einer Dependenz Montecassinos, durch Tassia, die Frau des Ratchis, setzt; und wieder findet sich diese Nachricht auch in Zusätzen zu den langobardischen Königslisten im Casinensis und im Cavensis. ${ }^{29}$ Ähnlich ist die Überlieferungslage bei der (ursprünglich von Paulus Diaconus berichteten) großen Schenkung Herzog Gisulfs II. sowie bei der Gründung von S. Sofia in Benevent (wo gleich zwei Zusätze im Casinensis die umstrittene Unterordnung unter Montecassino betonen). ${ }^{30}$ In allen Handschriften finden sich aber auch chronologische Bruchstücke, zum Beispiel eine ausführliche Aufstellung über die mehrfachen Herrschaftswechsel im Herzogtum Benevent im Laufe der 890er Jahre. ${ }^{31}$

Die meisten der in die Handschriften aufgenommenen kleinen Texte waren Gebrauchstexte. Die praktische Bedeutung von Papst- und Herrscherverzeichnissen und erklärenden Einschüben darin ist naheliegend. Verträge und Glossare konnten den rechtlichen Umgang mit der Außenwelt erleichtern oder den richtigen

\footnotetext{
${ }^{21}$ Hoffmann, Abtslisten (wie Anm. 5) 295-300.

${ }^{22}$ PoHL, Werkstätte (wie Anm. 1) 58-63 und 180-183.

${ }^{23}$ Le Liber pontificalis. Texte, introduction et commentaire, hg. von Louis DuCHESNE (Bibliothèque des Écoles Françaises d'Athènes et de Rome, ser. 2, 3). Paris 1886, 433 und 448f.

${ }^{24}$ Continuatio Casinensis (wie Anm. 14) 198-200.

${ }^{25}$ Cod. Casinensis 175, 561 .

${ }^{26}$ Chronicon Salernitanum (wie Anm. 12) c. 31-35, 33-37; PoHL, Werkstätte (wie Anm. 1) 61-63.

${ }^{27}$ Chronica monasterii Casinensis (wie Anm. 5) c. 1,7, $30 \mathrm{f}$.

${ }_{28}$ PoHL, Werkstätte (wie Anm. 1) 171; 183-185.

${ }^{29}$ Continuatio Casinensis (wie Anm. 14) 198-200.

${ }^{30}$ PoHL, Werkstätte (wie Anm. 1) 185-188 (mit Zusammenstellung aller Quellen).

31 PoHL, Werkstätte (wie Anm. 1) 190-195.
} 
Rat an die Herrschenden ermöglichen. Epitaphien und panegyrische Verse erinnerten nicht nur an bestimmte Personen, sondern konnten auch als Vorlage für ähnliche Dichtungen dienen. Viele der Texte konnten Material für Predigten bieten. Manche der recht drastischen Geschichten und Anekdoten eigneten sich auch dazu, an vornehme Laien weitererzählt zu werden, wenn diese im Kloster zu Gast waren. Bei solchen Gelegenheiten könnte auch der in allen drei Handschriften überlieferte parodierte Briefwechsel Karls des Großen und des Kaisers in Konstantinopel für die Unterhaltung von Nutzen gewesen sein. Darin kündigt der Byzantiner an, er werde Karl im Fall seiner Hilfe gegen den Kalifen 100.000 Goldstücke schicken - scias quia dirigo tibi aureorum centum milia. Karl antwortet: Scias quoniam dirigo tibi centum canes. ${ }^{32}$

Am reichsten ist die Cassineser Materialsammlung, was die Historiographie des 9. und 10. Jahrhunderts betrifft. Doch war das eine Geschichtsschreibung in Fragmenten, eine ,kleine Historiographie‘. In ständigen Brüchen und Neuansätzen wurden Serien von Ereignissen aufgebaut und wieder ausgeblendet. Schon der historiographische Anhang der Regelhandschrift von ca. 867 bestand aus einer Montage von Fragmenten, die in mehrfachen Rückblenden die wesentlichen Informationen zur Gegenwart und Vergangenheit der Abtei des heiligen Benedikt zusammenfügte. Erchempert schloss nicht daran an, sondern orientierte sich am Werk des Paulus Diaconus und seiner Fortsetzung. Nach ihm wurden nur mehr Bruchstücke niedergeschrieben, etwa für die Chronik von Capua. Das ist auffällig; immerhin wurden zugleich nördlich der Alpen selbst recht disparate Nachrichten durch konsequente Einordnung in ein annalistisches Schema in eine lineare Perspektive gebracht.

Methodisch ist dieser Charakter einer offenen Materialsammlung interessant. Er erlaubt Einblicke in die historiographische Werkstatt der Mönche von Montecassino. Die meisten Texte orientierten sich an der Chronologie der Fürsten; viele davon entstanden als Einschübe oder Nachträge in Fürstenkatalogen oder wurden darin kopiert. Anno-Domini-Datierungen waren zwar natürlich bekannt, aber offenbar unüblich. Freilich musste die Orientierung an den Fürstenlisten zu chronologischer Verwirrung führen. Bis zur Teilung des beneventanischen Prinzipats 849 konnte man sich relativ einfach orientieren, wie die Tabelle im Casinensis 175 zeigt: ${ }^{33}$ Einer Kolumne mit den Äbten stand eine mit den Fürsten von Benevent gegenüber. Ab Mitte des 9. Jahrhunderts komplizierten sich die Dinge; die Kolumnen vervielfältigten sich: Salerno, Sarazenen, Franken kamen dazu; später musste man vor allem mit Capua rechnen, auch byzantinische Statthalter spielten eine Rolle. Es ist kein Wunder, dass der komplizierteste Teil der beneventanischen Fürstenliste, von 892 bis 900, als einander in wenigen Jahren mehrere Mitglieder der Beneventaner Dynastie, Byzantiner, Spoletiner und schließlich Capuaner in der Herrschaft über Benevent abwechselten, in kommentierter Form in alle Handschriften kopiert wurde. Für Montecassino waren diese Herrschaftswechsel gar nicht gleichgültig; von fast allen dieser Fürsten erwarb man (oder konstruierte man nachträglich) weitreichende Privilegien und Besitzbestätigungen.

Ein einheitliches, lineares Geschichtsbild war in dieser Weise kaum zu gewinnen. Ebensowenig wurde ein geschlossenes, als solches dann weiter verbreitetes und verarbeitetes Kompendium angelegt. Stattdessen wurden die Texte in immer wechselnden Zusammenstellungen kopiert, verknappt, nachgetragen oder überarbeitet. Das mag unter anderem daran liegen, dass es keinen Anlass gab, alles Wesentliche in eine einzige Handschrift aufzunehmen. Es war wohl gerade die relativ reiche und alltägliche Schriftlichkeit, die es überflüssig machte, eine übersichtliche Darstellung der eigenen Geschichte anzufertigen und in einer einzigen Handschrift aufzubewahren. Schreiben in Montecassino fand im 9. und 10. Jahrhundert nicht in einer kargen Randzone der Schriftlichkeit statt, sondern in einer entwickelten Schriftkultur. Der fragmentarische Charakter der erhaltenen historiographischen Texte ist also nicht unbedingt Symptom des kulturellen Niedergangs, sondern eher die Spur eines alltäglichen Umgangs mit der Vergangenheit.

\footnotetext{
32 Alle drei Fassungen ediert in PoHL, Werkstätte (wie Anm. 1) 196f.

${ }^{33}$ Chronica sancti Benedicti Casinensis (wie Anm. 16) c. 22.
} 
